\title{
Effect of metal halide light source on hardness, water sorption and solubility of indirect composite material
}

\author{
Hiroyasu Koizumi ${ }^{1,4)}$, Hidetada Satsukawa ${ }^{1)}$, Naomi Tanoue ${ }^{2)}$, Tomohisa Ogino ${ }^{1)}$, \\ Minoru Nishiyama ${ }^{3,5)}$ and Hideo Matsumura ${ }^{1,4)}$ \\ Departments of ${ }^{1)}$ Crown and Bridge Prosthodontics and ${ }^{3)}$ Dental Materials, \\ Nihon University School of Dentistry, Tokyo, Japan \\ Divisions of ${ }^{4)}$ Advanced Dental Treatment and ${ }^{5)}$ Biomaterials Science, Dental Research Center, \\ Nihon University School of Dentistry, Tokyo, Japan \\ ${ }^{2)}$ Department of Specialized Dentistry, Nagasaki University Hospital of Medicine and Dentistry, \\ Nagasaki, Japan
}

(Received 10 August and accepted 15 September 2005)

\begin{abstract}
This study evaluates the effects of a metal halide light source on the post-polymerization properties of the Sinfony indirect composite material. Two polymerization systems were employed: the Hyper LII system, comprising a metal halide polymerization unit, and the Visio system, comprising two proprietary units designed for polymerizing the Sinfony composite. The composite material was polymerized for 60,120 or 180 $s$ with the LII system. As a control, the composite was polymerized for 15 min with the Visio system. Knoop hardness, water sorption and solubility were determined. The results were analyzed by Dunnett's T3 multiple comparison test $(P<\mathbf{0 . 0 5})$. Knoop hardness was greater for polymerization with the LII unit than for that with the Visio system. Water sorption was greater for polymerization with the Visio system than that with the LII unit. For polymerization with the LII unit for $180 \mathrm{~s}$, solubility was significantly reduced as compared with the Visio system. Within the limitations of the current experiment, it can be concluded that the metal halide unit exhibited better polymerizing performance for the composite material than the proprietary units. (J. Oral Sci. 47, 165-169, 2005)
\end{abstract}

Correspondence to Dr. Hiroyasu Koizumi, Department of Crown and Bridge Prosthodontics, Nihon University School of Dentistry, 1-8-13 Kanda-Surugadai, Chiyoda-ku, Tokyo 101-8310, Japan Tel: $+81-3-3219-8145$

Fax: +81-3-3219-8351

E-mail: koizumi@dent.nihon-u.ac.jp
Keywords: hardness; indirect composite; light source; solubility; water sorption.

\section{Introduction}

Over the last decade, the application of composites to indirect restorations and veneers for casting has increased considerably. This trend is mainly attributed to improvements in material properties (1-5). Indirect composite materials are polymerized with a light source, heat source, or both. Post-cure properties of the material are affected by the polymerizing system (6-10). The presence of unreacted monomer in the resin matrix negatively affects the properties of composite materials. Hardness, water sorption, and solubility in water are largely related to the conversion of monomers incorporated into composites. To ensure properties which confer durability in vivo, it is vital to increase monomer conversion in polymerization of the material.

A laboratory light-polymerizing unit equipped with two metal halide lamps has been developed (11). The apparatus demonstrates excellent performance compared with other polymerizing systems $(7,9,12-15)$. In addition, a previous study showed that application of two different light sources improves the properties of indirect composite materials (16). Manufacturers of composite material typically recommend the use of proprietary polymerizing systems for laboratory work. However, there is little information on the post-cure properties of indirect composites polymerized with nonproprietary systems. 
The present study evaluates the performance of a laboratory-curing unit equipped with a metal halide light source in polymerization of an indirect composite.

\section{Materials and Methods}

A composite material designed for inlays, onlays and restoration veneers (Sinfony, 3M ESPE, Seefeld, Germany) was selected. The composite is composed of $50-55 \mathrm{wt} \%$ aliphatic and cycloaliphatic monomers, and $45-50 \mathrm{wt} \%$ aluminum glass and silica fillers with a particle size distribution of $0.5-0.7 \mu \mathrm{m}(3,17)$. Of the four shades for enamel material, the E3 shade was used for property testing.

Two laboratory light polymerizing systems were used. One system is a high-intensity polymerizing unit equipped with two metal halide lamps (Hyper LII, Toho Dental Products, Saitama, Japan; LII) (11). The other system consists of two light polymerizing units (Visio Alfa and Visio Beta Vario, 3M ESPE). The Visio Alfa unit is equipped with a halogen lamp whereas the Visio Beta Vario unit is equipped with four fluorescent tubes. The Visio units are recommended by the manufacturer for polymerizing the Sinfony composite, and thus the combination of the two units was considered as the proprietary system in the current study. Details on the material, polymerizing systems and polymerization conditions are summarized in Table 1 . To assess the curing performance, specimens were prepared with either the LII unit or the Visio system. Knoop hardness, water sorption and solubility in water were determined. The distance from the light source to the specimen surface was approximately $10 \mathrm{~mm}$ for the Visio Alfa unit, $40 \mathrm{~mm}$ for the Visio Beta Vario unit and $80 \mathrm{~mm}$ for the LII unit.

\section{Knoop hardness}

The Sinfony composite was added to a glass tube (10 $\mathrm{mm}$ in diameter $\times 10 \mathrm{~mm}$ ), which was then covered with a glass plate (0.12 - $0.17 \mathrm{~mm}$ thickness). Polymerization was performed as follows: 1) Visio Alfa for $15 \mathrm{~s}$ and Visio Beta Vario for 15 min (Group 1, control); 2) LII for $60 \mathrm{~s}$ (Group 2); 3) LII for 120 s (Group 3); and 4) LII for 180 s (Group 4). After polymerization, the glass plates were removed and each specimen was ground under running water with a series of silicon-carbide $(\mathrm{SiC})$ papers, and polished with felt and alumina $(0.3 \mu \mathrm{m}$, Baikalox $0.3 \mathrm{CR}$, Baikowski International, Charlotte, NC, USA). Specimens were stored in water at $37^{\circ} \mathrm{C}$ for $24 \mathrm{~h}$ and Knoop hardness number (KHN) was determined at the top surface using a universal indenter (Micro Hardness tester, Akashi., Yokohama, Japan) with application of a $25 \mathrm{~g}$ load for $30 \mathrm{~s}$.

\section{Water sorption and solubility in water}

Material paste was added to a plastic mold having a diskshaped opening $(15 \mathrm{~mm}$ in diameter $\times 1 \mathrm{~mm})$ covered with two pieces of transparent glass plate. The material was polymerized as described for hardness testing. After polymerization, specimens were removed from the mold, ground with abrasive paper and wet-polished with aluminum oxide. Water sorption and solubility in water were determined according to the method described in ISO 10477 (18).

\section{Statistical analysis}

Specimens were divided into four groups according to polymerizing system and total exposure time; Group 1 (Visio), Group 2 (LII, 60 s), Group 3 (LII, 120 s) and Group 4 (LII, $180 \mathrm{~s}$ ). The mean value and standard deviation of five specimens were calculated for each of the three property tests. The results were analyzed by the Levene test and by Dunnett T3 multiple comparison (SPSS software Version 12.0.2 for Windows, SPSS, Chicago, IL, USA) with statistical significance set at $P=0.05$. The results for

Table 1 Material and light-polymerizing units assessed

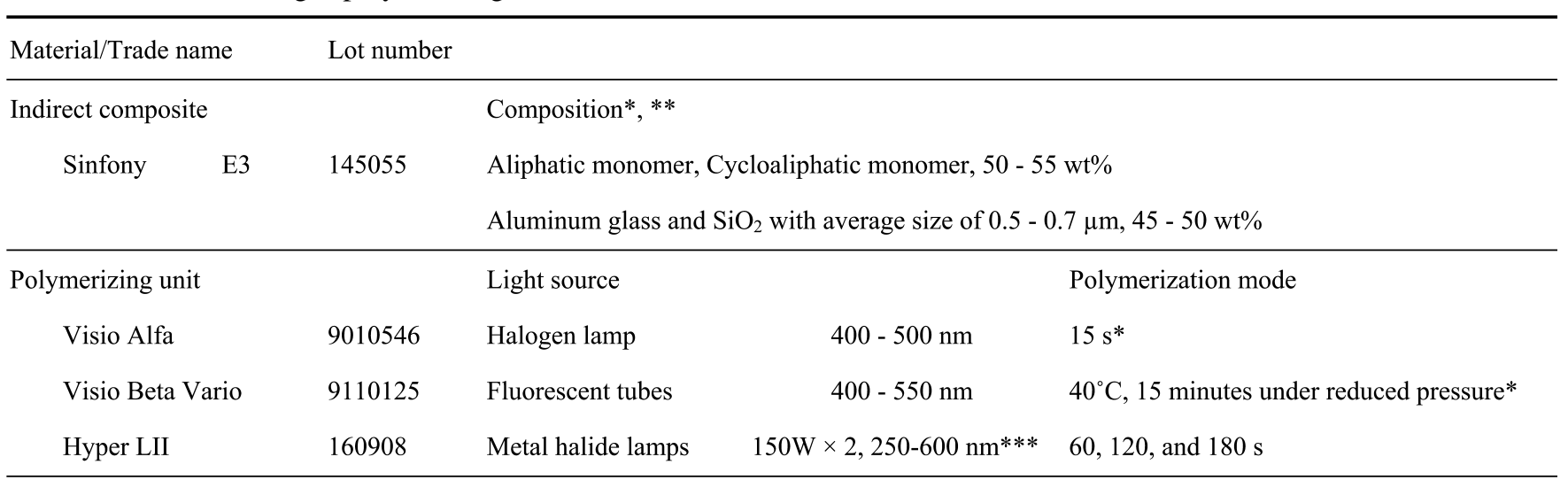

*Kakaboura et al. (3), ${ }^{* *}$ Göhling et al. (17), ***Matsumura et al. (11) 
Group 1 (control) were quoted from a previous paper (16).

\section{Results}

Levene tests run on the three property tests gave the following p-values; 0.386 for KHN, 0.984 for water sorption, and 0.034 for solubility. Of the three tests, solubility in water did not exhibit homoscedasticity. All three test results were therefore analyzed by Dunnett T3 multiple comparison.

Results for hardness testing are shown in Fig. 1. KHN of the Sinfony composite polymerized with the Visio system (24.8, category A) was statistically inferior to that recorded with the LII unit (31.4 - 34.5, category B). Extension of the exposure period with the LII unit did not improve hardness of the composite material $(P>0.05)$ within the range of $60-180 \mathrm{~s}$.

Water sorption in the four groups is presented in Fig. 2. The Sinfony composite exhibited maximal sorption when the material was polymerized with the Visio system $\left(25.6 \mu \mathrm{g} / \mathrm{mm}^{3}, P<0.05\right)$. The three groups polymerized with the LII unit showed water sorption ranging from 12.7 to $13.6 \mu \mathrm{g} / \mathrm{mm}^{3}$. There were no statistically significant differences between the LII groups (category D), but all LII values were significantly lower than those obtained for the Visio system (category C).

The solubility of the Sinfony composite in water is summarized in Fig. 3. The Sinfony composite had greater water sorption when polymerized with the Visio system

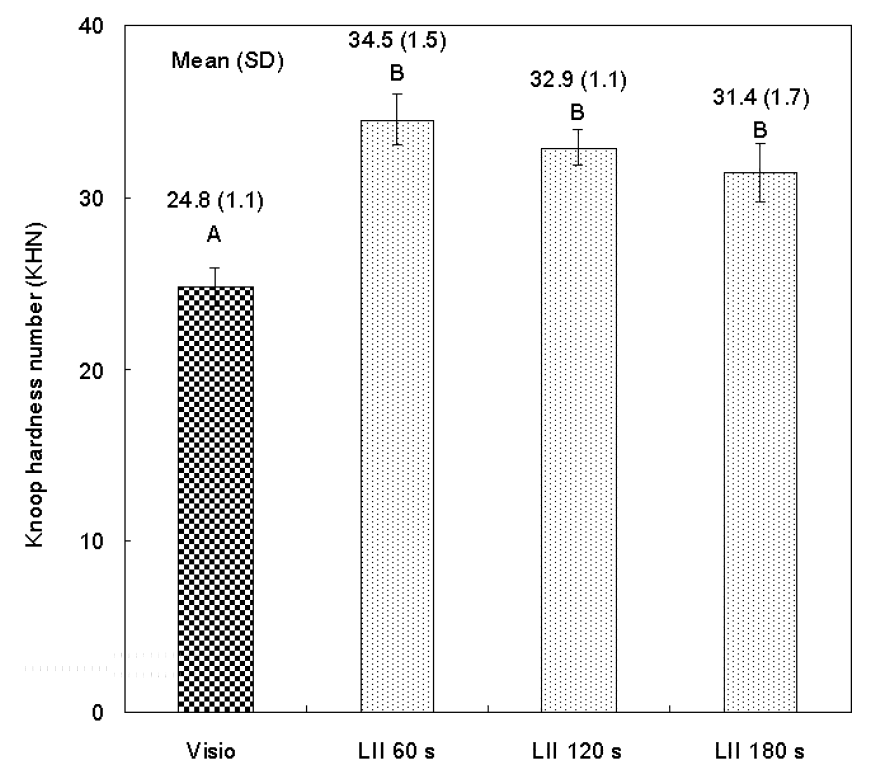

Fig. 1 Hardness testing results for the Sinfony composite. Standard deviations in parentheses. Identical letters indicate that values are not statistically different $(P>$ $0.05)$. The value for the Visio system was quoted from Satsukawa et al., 2005 (16). $\left(3.1 \mu \mathrm{g} / \mathrm{mm}^{3}\right)$ than when polymerized with the LII unit for $180 \mathrm{~s}\left(0.9 \mu \mathrm{g} / \mathrm{mm}^{3}, P<0.001\right)$. No significant differences in solubility were identified among the category E groups (Visio, LII $60 \mathrm{~s}$, and LII $120 \mathrm{~s}$ ) or among the category F groups (LII $60 \mathrm{~s}$, LII $120 \mathrm{~s}$, and LII $180 \mathrm{~s}$ ).

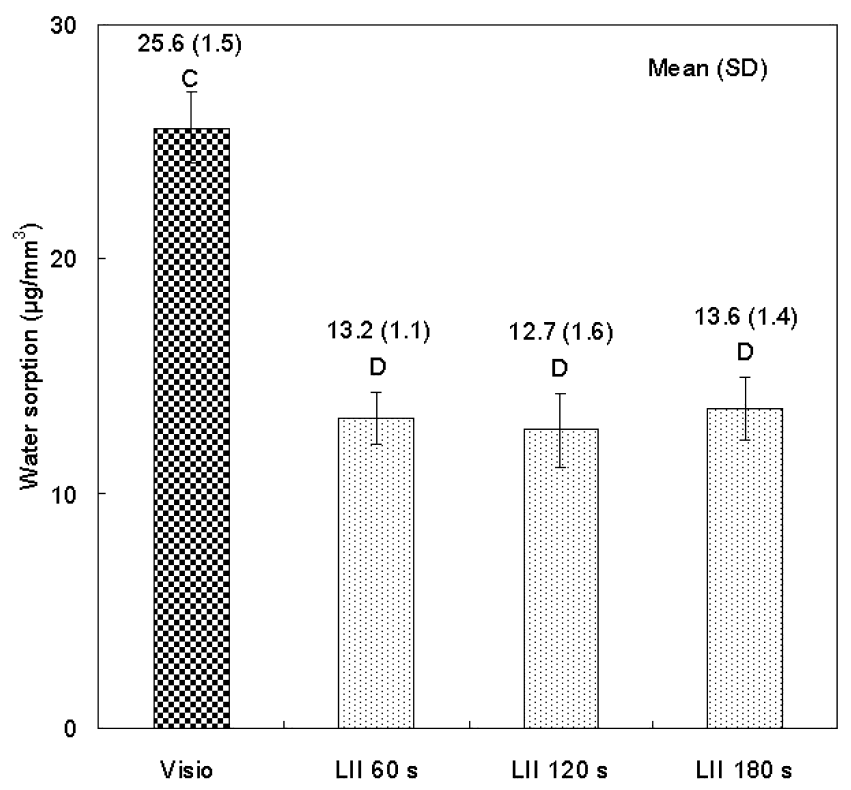

Fig. 2 Water sorption of the Sinfony composite. Standard deviations in parentheses. Identical letters indicate that values are not statistically different $(P>0.05)$. The value for the Visio system was quoted from Satsukawa et al., 2005 (16).

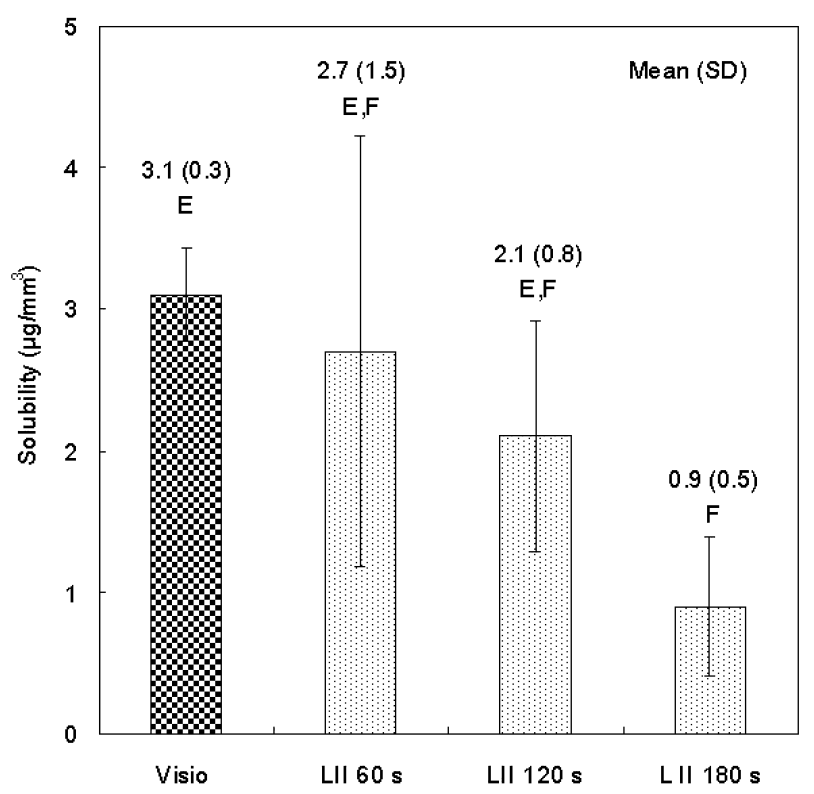

Fig. 3 Solubility of the Sinfony composite in water. Standard deviations in parentheses. Identical letters indicate that values are not statistically different $(P>0.05)$. The value for the Visio system was quoted from Satsukawa et al., 2005 (16). 


\section{Discussion}

The post-cure properties of composite materials are influenced by filler loading, type of monomers in the matrix, polymerizing apparatus, shade of material and other factors (8-13). Since an appropriate volume is required for determination of Knoop hardness, the specimen thickness for hardness testing in the current study was set to $10 \mathrm{~mm}$. In addition, the location of testing was set at the polished superficial layer, at which point exposed light energy was considered to be sufficient. The KHN of the Sinfony composite polymerized with the Visio system was 24.8 , even though the material was exposed for more than 15 min. In contrast, the KHN of the composite polymerized with the LII unit was 31.4 or more, despite a curing time of $3 \mathrm{~min}$ or less. Significant difference in hardness testing results suggests that there is a room for improvement in emission energy for the Visio system especially for the Visio Beta Vario unit, although neither light intensity nor emission energy were determined in this study. A previous study (16) concluded that a polymerizing unit with two halogen lamps and a metal-halide lamp is effective for polymerizing the Sinfony composite. The present study shows that the combination of a lightpolymerizing unit with two metal-halide lamps was more effective for polymerizing the Sinfony composite than the Visio system.

Water sorption of composite material is affected by polarity and conversion of monomers in the matrix, polymerization mode and other factors $(6,11,13,19,20)$. The Sinfony composite contains aliphatic and cycloaliphatic monomers, which do not contain hydrophilic groups (3). It is therefore difficult to explain the considerable water sorption of the Sinfony composite based solely on monomer polarity. Since the water sorption of the Sinfony composite was significantly reduced when the material was polymerized with the LII unit, the results of water sorption support the notion that the intensity of the Visio Beta unit is insufficient to polymerize the Sinfony composite.

Dunnett T3 analysis demonstrated that solubility of the Sinfony composite was significantly greater when polymerized with the Visio system than when polymerized with the LII unit for $180 \mathrm{~s}$. The results indicate that the amount of unpolymerized monomer remaining in the matrix or eluted monomer is greater for polymerization with the Visio system as compared with the LII unit for $180 \mathrm{~s}$.

In the present study, specimens polymerized with the LII unit exhibited better overall properties than those polymerized with the Visio system, even though the distance between the specimen and light source is greater for the LII unit. This is probably due to difference in emission energy of light sources. However, in laboratory polymerization of indirect composites, the distance between the material and light source should be reduced as much as possible. Within the limitations of this study, it can be concluded that for polymerization of the Sinfony composite, the Hyper LII metal halide unit is superior to the proprietary curing units in terms of the post-cure properties.

\section{Acknowledgments}

This study was supported in part by a Grant-in-Aid for Scientific Research B(2) 16390566 (2004-2005) from the Japan Society for the Promotion of Science (JSPS), by a Grant-in-Aid for Young Scientists B(2) 16791210 (20042005) from the Ministry of Education, Culture, Sports, Science and Technology of Japan (MEXT-Japan), and by the Sato Fund (2004) of Nihon University School of Dentistry.

\section{References}

1. Young HL, Suzuki S (1999) Wear of composite resin inlays and antagonistic enamel. Am J Dent 12, 47-50

2. Stober T, Gilde H, Lenz P (2001) Color stability of highly filled composite resin materials for facings. Dent Mater 17, 87-94

3. Kakaboura A, Rahiotis C, Zinelis S, Al-Dhamadi YA, Silikas N, Watts DC (2003) In vitro characterization of two laboratory-processed resin composites. Dent Mater 19, 393-398

4. Keski-Nikkola MS, Alander PM, Lassila LV, Vallittu PK (2004) Bond strength of Gradia veneering composite to fibre-reinforced composite. J Oral Rehabil 31, 1178-1183

5. Lehmann F, Eickemeyer G, Rammelsberg P (2004) Fracture resistance of metal-free composite crownseffects of fiber reinforcement, thermal cycling, and cementation technique. J Prosthet Dent 92, 258264

6. Kildal KK, Ruyter IE (1997) How different curing methods affect mechanical properties of composites for inlays when tested in dry and wet conditions. Eur J Oral Sci 105, 353-361

7. Tanoue N, Matsumura H, Atsuta M (2000) Comparative evaluation of secondary heat treatment and a high intensity light source for the improvement of properties of prosthetic composites. J Oral Rehabil 27, 288-293

8. Dietschi D, Marret N, Krejci I (2003) Comparative efficiency of plasma and halogen light sources on composite micro-hardness in different curing conditions. Dent Mater 19, 493-500 
9. Tanoue N, Atsuta M, Matsumura H (2003) Properties of a new photo-activated composite polymerized with three different laboratory photo-curing units. J Oral Rehabil 30, 832-836

10. Da Fonte Porto Carreiro A, Dos Santos Cruz CA, Vergani CE (2004) Hardness and compressive strength of indirect composite resins: effects of immersion in distilled water. J Oral Rehabil 31, 1085-1089

11. Matsumura H, Tanoue N, Atsuta M, Kitazawa S (1997) A metal halide light source for laboratory curing of prosthetic composite materials. J Dent Res 76, 688-693

12. Tanoue N, Matsumura H, Atsuta M (1998) Curing depth of four composite veneering materials polymerized with different laboratory photo-curing units. J Oral Rehabil 25, 348-352

13. Tanoue N, Matsumura H, Atsuta M (1998) Properties of four composite veneering materials polymerized with different laboratory photo-curing units. J Oral Rehabil 25, 358-364

14. Tanoue N, Matsumura H, Atsuta M (1999) Curing depth of prosthetic composite materials polymerized with their proprietary photo-curing units. J Oral
Rehabil 26, 594-599

15. Tanoue N, Matsumura H, Atsuta M (1999) Effectiveness of polymerization of a prosthetic composite using three polymerization systems. J Prosthet Dent 82, 336-340

16. Satsukawa H, Koizumi H, Tanoue N, Nemoto M, Ogino T, Matsumura H (2005) Properties of an indirect composite material polymerized with two different laboratory polymerizing systems. Dent Mater J 24, 377-381

17. Göhring TN, Gallo L, Lüthy H (2005) Effect of water storage, thermocycling, the incorporation and site of placement of glass-fibers on the flexural strength of veneering composite. Dent Mater 21, 761-772

18. International Organization for Standardization (1992) Dentistry - Polymer-based crown and bridge materials. ISO 10477, 1st ed, Geneve

19. Marcovich NE, Reboredo MM, Aranguren MI (1999) Moisture diffusion in polyester-woodflour composite. Polymer 40, 7313-7320

20. Hori M, Yoshida E, Hashimoto M, Kaga M, Sano $\mathrm{H}$, Oguchi $\mathrm{H}$ (2004) In vitro testing of all-in-one adhesives as fissure sealants. Am J Dent 17, 177181 